\title{
Erratum to: Philosophy and Oscar Wilde
}

Michael Y. Bennett

\section{Erratum to:}

Michael Y. Bennett, Philosophy and Oscar Wilde

DOI 10.1057/978-1-137-57958-4

The publisher has made metadata changes that do not affect the content.

The updated original online version for this book can be found at: DOI 10.1057/978-1-137-57958-4

Michael Y. Bennett

University of Wisconsin-Whitewater

Department of Languages and Literatures

Stoughton, Wisconsin, USA 